\title{
LA ENFERMEDAD COMO ELEMENTO DE DISCRIMINACIÓN SOCIAL EN EL NORTE DE PORTUGAL, A FINALES DEL SIGLO XIX Y PRINCIPIOS DEL XX
}

\author{
Alexandra ESTEVES \\ Recibido: 01/07/2012 \\ Aceptado: 19/09/2012
}

RESUMEN: El objetivo de nuestra comunicación es entender las razones de la estigmatización que cayó sobre los hombres y mujeres que se vieron afectados por ciertas enfermedades, por lo que dicha discriminación se materializa por la estructura social y los mecanismos desarrollados por el Estado para el tratamiento y la protección de estos los pacientes. Esto no deja de ser un síntoma de la persistencia de algunos "fantasmas" que continuaron a participar de ciertas enfermedades, para lo cual la comunidad médica en sí misma no había encontrado todavía una explicación.

PALABRAS CLAVE: enfermedad, discriminación, Norte de Portugal.

ABSTRACT: The purpose of our communication is to understand the reasons for the stigmatization that fell upon men and women who were affected by certain diseases, so that such discrimination was materialized by social structure and the mechanisms developed by the State for treatment and protection of these patients. This does not fail to be a symptom of the persistence of some "ghosts" who continued to engage certain diseases, for which the medical community itself had not yet found an explanation.

KEYWORDS: diseases, discrimination, North of Portugal.

La historia ha demostrado que la enfermedad, especialmente cuando alcanza dimensiones endémicas y pandémicas, puede constituir una especie de válvula de escape para los comportamientos instintivos e incluso grotescos, desde el punto de vista de la ética y la moral judeo-cristiana, que, a su vez, ponen de manifiesto el componente social de la enfermedad.

Con el pretexto de una supuesta cura de desinfección o erradicación de enfermedades, se cometieron auténticas limpiezas étnicas, las minorías fueron perseguidas y maltratadas, naciones y pueblos humillados y oprimidos. La historia nos muestra, por lo tanto, que la enfermedad sirvió como pretexto para la discriminación y, a veces, para la violencia social. Los casos más visibles de este 
tipo de discriminación fueron el resultado de acciones promovidas por los propios Estados, que, incapaces de luchar contra la enfermedad, convirtieron a ciertos sectores de la sociedad en chivos expiatorios. El objetivo predilecto eran los grupos socialmente desfavorecidos y marginados, especialmente los pobres, mendigos y vagabundos, prostitutas, e incluso las mujeres en general. Tomemos el caso de los judíos, perseguidos en algunas partes de Europa, acusados de propagar la peste negra por el envenenamiento de pozos, de las prostitutas, un símbolo de la conducta sexual promiscua, culpadas de la propagación de la sífilis, los pobres, considerados como transmisores una serie de enfermedades y responsabilizados de las enfermedades endémicas, siendo, por otra parte, objeto de las miradas de desaprobación y desconfianza de una sociedad burguesa en desarrollo desde finales del siglo XVIII ${ }^{1}$.

Paradójicamente, en otro aspecto, se observa la existencia de enfermedades, que, en lugar de generar rechazo, a pesar de ser muy contagiosas, despertaron sentimientos cercanos al ámbito de la mística y del romanticismo. ${ }^{2}$ Eso es lo que ocurrió, por ejemplo, con tuberculosis, asociada a artistas e intelectuales, a pesar de que es una enfermedad que remite a la idea de discriminación y alejamiento. De este modo, una de las preguntas se pueden formular está relacionada, precisamente, con la actitud de la sociedad frente a la enfermedad: por qué, en algunos casos, se culpa y se persigue a los presuntos responsables de su aparición y propagación, mientras que en otros opta por la solidaridad y la caridad, haciendo que la enfermedad, o mejor dicho, su tratamiento, sea un elemento de cohesión de la comunidad?

$\mathrm{Si}$, en algunas circunstancias, la enfermedad potencia mecanismos de solidaridad, en otros genera violencia, especialmente cuando la gente no entiende ni acepta las medidas sanitarias impuestas por las autoridades para controlar y prevenir la propagación de enfermedades contagiosas. La región del Miño, en el norte de Portugal, que limita con la provincia española de Galicia, se vio afectada por el cólera en el siglo XIX. Para combatir la epidemia, las autoridades levantaron un cordón sanitario, con influencias directas en la vida cotidiana de la población, en particular respecto de sus movimientos. Sin embargo, por entender que esta

\footnotetext{
${ }^{1}$ Véase Duffin, J. (2000). History of Medicine. London: Macmillan Press, pp. 139-167.

${ }^{2}$ Es el caso de la tuberculosis en Portugal en los últimos años del siglo XIX. Véase FerREIRA, M. (2005). A doença do peito. Contributo para o estudo histórico da tuberculose. Porto: Faculdade de Letras do Porto, pp. 20-21.
} 
medida perjudicaría a sus intereses económicos y que sus hábitos tradicionales se ponían en tela de juicio, se negó a cumplirla rebelándose contra sus autores ${ }^{3}$.

De hecho, son los disturbios, motines y levantamientos, motivados por la introducción de medidas sanitarias, las mejor reflejadas por la historiografía, consideradas como un paradigma de la relación entre la aparición de la violencia social y la enfermedad. Este hecho es el resultado, por supuesto, de la mayor visibilidad de aquellos modos de protesta, del elevado número de personas implicadas y de la dureza de la reacción de las autoridades.

No es nuestro propósito estudiar estas situaciones de violencia colectiva, que tuvieron lugar en varias regiones de Europa, especialmente en el siglo XIX, ni las medidas para combatir la enfermedad, solo tenemos la intención de mostrar la perspectiva del enfermo y, sobre todo, analizar la forma en que la enfermedad le convirtió en objeto de discriminación social.

El prejuicio asociado a una enfermedad determinada se producía no sólo por las conductas y prácticas que podrían estar la génesis de la enfermedad, aunque erróneamente entendida por la sociedad, como la sífilis o la tiña, sino también por la actitud del propio enfermo, condicionada por la enfermedad que padecía, como ocurrió con los enfermos mentales, o por las huellas físicas inconfundibles, como ocurrió con las víctimas de la viruela, o incluso por la apariencia física, como ocurrió, por ejemplo, con la tuberculosis. Por supuesto no podemos negar el miedo al contagio, tal vez el más pernicioso.

El estigma que recaía sobre los pacientes era evidente en varios niveles, como señalaremos, y era originado, aunque de forma involuntaria, por el Estado y las autoridades sanitarias, los cuales, a través de sus medidas, suscitaban actitudes de rechazo. La incapacidad para conocer el origen de la enfermedad sería, por supuesto, uno de los factores que explican el doble sentimiento de temor y discriminación ${ }^{4}$.

${ }^{3}$ Sobre el alcance del cólera en el Alto Miño, en el siglo XIX y las medidas impuestas por las autoridades sanitarias y administrativas, consúltese EsTEVES, A.; ARAÚJO, M. (2010). «Cholera in the Portuguese region of Alto Minho in the second half of the nineteenth century: epidemic outbreaks, treatment and behaviours». Hygiea Internationalis: An Interdisciplinary Journal for the History of Public Health, , vol. 9, n. 1, pp. 183-198 e Esteves, A. Assistência às vítimas de cólera no Alto Minho na primeira metade do século XIX. (cd.rom).

${ }^{4}$ Léase CAPLAN, A. (2001). "The concepts of health, illness, and disease". En BYNUM, W. F.; PORTER, Roy. Companion encyclopedia of the history of medicine. vol. 1. London and New York. pp. 233-248. 
Esto se manifestaba en la vida cotidiana, especialmente a través del uso de expresiones ofensivas. Uno de los insultos más utilizados en el norte de Portugal en el siglo XIX, era "que tiene sífilis", lo que significaba que se estaba ante alguien con una conducta sexual reprobable, alcanzando aún una dimensión mayor si se trataba de una mujer, pues sería sinónimo de desvergonzada, dada la elevada incidencia de enfermedades venéreas entre las prostitutas. El 23 de septiembre de 1856, el gobernador civil de Viana do Castelo informó al administrador del mismo concejo (município) de la infección por sífilis de militares, como resultado del contacto con prostitutas infectadas, indicando lo siguiente:

Tendo o brigadeiro comandante de infantaria $n^{\circ} 3$ sollicitado providencias desta repartição acerca das meretrizes, que, segundo consta da participação do hospital regimental, tem infeccionado de vírus sypphitus, algumas praças d'aquelle corpo, queira Vossa Senhoria adoptar as medidas convenientes para que as meretrizes que não são naturaes deste concelho sejam mandadas para fora do mesmo, e para que as doentes marchem para o hospital de S. Marcos de Braga, praticando as mais diligencias que são d'uso em cazos semelhantes ${ }^{5}$.

Asociadas al pecado, estas enfermedades eran consideradas vergonzosas para los afectados, lo que explica su inclusión en la lista de ofensas. Aun más, se trataba de una enfermedad que, además de causar un inmenso sufrimiento, dañaba diferentes partes del cuerpo y deformaba otras, y que requería un tratamiento a base de mercurio, igualmente doloroso. ${ }^{6}$

En 1932, Henrique Jorge Niny destacaba que "la enfermedad ha sido injustamente acusada de vergonzosa", siendo una infección que afectaba indistintamente a hombres, mujeres y niños a través de diferentes formas de contagio. Detallaba, sin embargo, que en Portugal la situación era particularmente difícil para las mujeres, que, dominadas por lo que él llama "los viejos y mohosos

5 AHGCVC, Registo geral de Julho de 1856 a 31 de Dezembro, n. ${ }^{\circ} 1.10 .3 .3$, sin paginar. El Hospital de S. Marcos, en Braga, acogía enfermos de sífilis en enfermerías destinadas a este tratamiento y que se abrían dos veces al año. Léase a este propósito CASTRO, M. (1993). «Assistência no Hospital de S. Marcos na 2. ${ }^{a}$ metade do século XVII a cerca de 1710» Revista Cultural Bracara Augusta, n. ${ }^{\circ}$ 44, pp. 45-73. Sobre la sífilis, léase JÜTTE, R. (1996). «Syphilis and confinement. Hospitals in early Modern Germany». En JunKer, D.; MatTeM, S. D (eds.), Institutions of confinement, hospitals, asylums and prisons in Western Europe and North America 1500-1950. Cambridge: Cambridge University Press, pp. 97-115.

${ }^{6}$ Véase Lindemann, M. (2002). Medicina e sociedade no início da Europa moderna. Lisboa: Replicação, pp. 55-58. 
prejuicios de una errónea y falsa moral"7, abandonaban el tratamiento o simplemente no se trataban, contribuyendo, de ese modo, a la propagación de la enfermedad ${ }^{8}$.

La sífilis, en particular, fue profundamente discutida durante el siglo XIX ${ }^{9}$ tratándose de una enfermedad transmitida por vía sexual, patente por la presencia de lesiones de la piel extraordinariamente contagiosas. Aunque discutible, se cree que llegó a Europa procedente del continente americano, habiéndose transformado rápidamente en un arma arrojadiza entre las naciones: para los franceses era el "mal" italiano, para los italianos era el "mal" francés, para los africanos, japoneses e indios era "mankabassam", es decir, el mal portugués. ${ }^{10}$ En Portugal, pronto fue conocida como "gálico", asociada, por lo tanto, a tierras francesas. En el siglo XIX, crece el recelo hacia esta enfermedad. Considerada un ataque a la familia y la sociedad en general, la sífilis exigía una intervención más eficaz por parte de las autoridades estatales. Estas, a su vez, dirigen su atención hacia un grupo concreto considerado el principal vehículo de transmisión: las prostitutas ${ }^{11}$.

En Portugal, en el siglo XIX, la prostitución no estaba prohibida, pero se procuraba una higienización, dada su relación con la propagación de enfermedades venéreas en los individuos que estaban en edad fértil. El Código Administrativo de 1836 previa una cierta permisión con la prostitución, no con el fin de facilitarla, sino más bien para controlarla. Artículo 109. § 6 de este documento prohibía la permanencia de las prostitutas en los más concurridos espacios públicos, como iglesias, plazas y escuelas, mientras el gobierno no "publicase reglamentos especiales". ${ }^{12}$ De este modo, la práctica de la prostitución quedaba restringida a las zonas más apartadas de las localidades, como un intento de evitar el escándalo o el mal ejemplo, teniendo el administrador del concejo que velar por el cumplimiento de las normas establecidas.

${ }^{7}$ Compárese NinY, H. (1932). «Combate à sífilis». Boletim do Instituto de Criminologia, vol. XVI, p. 63.

${ }^{8}$ Véase NiNY, H. (1932). «Combate à sífilis». Boletim do Instituto de Criminologia, vol. XVI, p. 62.

9 Compárese Moulin, Anne-Marie; Delort, R. «Sífilis - mal americano?». En Duby, G. (introducción), Amor e Sexualidade no Ocidente. Lisboa: Terramar, pp. 295-309.

${ }^{10}$ Compruébese Harrison, M. (2009). Disease na the Modern World. 1500 to the present day. Cambridge: Polity Press, p. 35.

11 Véase sobre este tema BRANDT, A. (2001). «Sexuallity transmitted diseases». En BYNUM, W.

F.; PORTER, R. Companion encyclopedia of the history of medicine, vol. 1..., pp. 562-565.

${ }^{12}$ Véase Código Administrativo Portuguez. (1838). Lisboa: Imprensa Nacional, p. 64; 72. 
A lo largo del siglo XIX, el administrador del concejo debía proceder al registro de las prostitutas residentes en la localidad bajo su jurisdicción, para que pudieran ser sometidas a inspección sanitaria. Las que faltasen sin justificación a la convocatoria de las autoridades podían ser castigadas. ${ }^{13}$ El Código Administrativo de 1842 sigue la misma línea, atribuyendo al administrador del concejo la responsabilidad de la vigilancia de las prostitutas. ${ }^{14}$

Analizando el Regulamento Policial das Toleradas do Concelho de Braga, con fecha de 1871, se comprueba que eran consideradas prostitutas o toleradas a las mujeres que, abiertamente, hacían de la prostitución su modo de vida, frecuentando casas relacionadas con esta ocupación, así como las mujeres que no teniendo modo conocido de ganarse la vida, recibían en sus domicilios la visita frecuente de hombres o que cohabitan con soldados, sin demostrar que estaban casados con ellas. ${ }^{15}$ La vigilancia policial de las toleradas tenía básicamente dos propósitos: por un lado vigilar el comportamiento de las prostitutas, reprimir los excesos y ayudar a aquellas que deseasen abandonar la prostitución, y, por otro, garantizar el cumplimiento de las normas sanitarias para impedir la propagación de la sífilis y otras enfermedades venéreas. ${ }^{16}$

La vigilancia de las toleradas estaba a cargo del administrador del concejo, de un agente de policía sanitaria y de un empleado de la administración. El servicio de salud era competencia de un médico designado por el gobernador civil, que estaba encargado de hacerles el reconocimiento semanal en el dispensario de la ciudad o en su propio domicilio, de darles instrucciones sobre higiene íntima y personal, sobre la limpieza de la ropa y de la casa. Si alguna se encontraba infectada con sífilis u otra enfermedad contagiosa, se la llevaba de inmediato al hospital, acompañada de una guía emitida por el médico. Al final de cada mes, el doctor tenía que presentar una lista con el número de toleradas examinadas, indicando los casos de sífilis detectados.

${ }^{13}$ Sobre la prostitución en el siglo XIX, léase PAIS, J. (2006). A prostituição e a Lisboa boémia do século XIX a inícios do século XX. Lisboa: Âmbar, pp. 43-62.

14 Consultar Código Administrativo Portuguez. (1849). Lisboa: Imprensa Nacional, p. 105. Liberato, I. (2002). Sexo, Ciência, Poder e exclusão social. A tolerancia da prostituição em Portugal (1841-1926). Lisboa: Livros Brasil, p. 175.

${ }^{15}$ Regulamento policial das toleradas no concelho de Braga. (1981). Braga, pp. 1-2.

${ }^{16}$ Sobre este tema, léase LiBERATO, M. (2000). «Da tolerância da prostituição à exclusão social da prostituição». En VAZ, M.; Relvas, E. Exclusão na História. Actas do Colóquio Internacional sobre Exclusão Social. Lisboa: Celta, pp. 53-58. 
Al agente de la policía sanitaria le competía presentar al médico inspector la relación de las toleradas sujetas a observación, llevarlas al hospital o al lugar donde serían examinadas, siendo avisadas con 24 horas de antelación de la obligación de presentarse o que podrían ser visitadas. Las que no cumpliesen con esta disposición podían ser puestas bajo custodia (vigilancia), serían multadas o castigadas con ocho días de detención. Casi todas las prostitutas eran consideradas toleradas, con excepción de las menores de 17, aunque se procurase, antes de su registro, disuadirlas de la prostitución ${ }^{17}$.

Esta actitud de tolerancia en relación con la prostitución esconde una actitud discriminatoria y aislacionista, dictada principalmente por razones sanitarias. Hay una clara necesidad de identificar a las prostitutas para poder controlarlas y aislarlas como forma de evitar la propagación de enfermedades venéreas y proteger la salud pública. Esta afirmación es corroborada por la obligación de que existiese, en el municipio, un libro de registro de las casas de tolerancia o colegios, es decir, casas gobernadas por una mujer que tenía bajo su dirección a varias chicas dedicadas a la prostitución. Estos colégios, además de poder ser inspeccionados, sin previo aviso, por las autoridades policiales, debían ser visitados regularmente por el médico, para comprobar que la casa tenía mobiliario, utensilios y los recursos indispensables para su limpieza.

Estas mujeres podían residir en cualquier punto de la ciudad, excepto cerca de los templos, de las escuelas, de las reparticiones públicas, de los cuarteles o dentro de las hospederías $[\ldots]^{18}$. Las ventanas de sus casas tenían que estar protegidas por persianas, no podían permanecer durante mucho tiempo en espacios públicos, ni estar en la ventana o a la puerta de su casa de "forma indecente" 19 . No podían cambiar de residencia sin comunicárselo al administrador del concejo, ni incluso salir de la localidad donde estaban registradas bajo pena del pago de una multa.

Otra medida claramente discriminatoria, patente en el Regulamento das Toleradas da cidade de Braga, disponía que, en el caso de ser consideradas incurables, no podrían residir en el municipio de Braga, debiendo inernarse en un hospital o institución que las acogiese ${ }^{20}$.

Las prostitutas no siempre cooperaban con las autoridades. La prensa daba cuenta de las dificultades experimentadas por las autoridades administrativas para

\footnotetext{
${ }^{17}$ Regulamento policial das toleradas no concelho de Braga..., p. 5

${ }^{18}$ Regulamento policial das toleradas no concelho de Braga ..., p. 11

${ }^{19}$ Regulamento policial das toleradas no concelho de Braga ..., p. 12

${ }^{20}$ Regulamento policial das toleradas no concelho de Braga..., p. 11
} 
su control. Las escaramuzas entre las prostitutas, vecinos y transeúntes eran frecuentes y muchas de ellas terminaban la noche detrás las rejas, por considerarse que su conducta había contribuido al estallido de verdaderos disturbios ${ }^{21}$. Su presencia, sobre todo al caer la noche, era vista como un estorbo que impedía que algunas familias saliesen a la calle ${ }^{22}$. En el caso particular de Viana do Castelo, existían algunos lugares asociados con "mujeres perdidas". Grupos de prostitutas se reunían en el atrio de la capilla de Nuestra Señora de la Agonía o debajo del arco de la iglesia de Nuestra Señora de la Victoria y también en una taberna cerca del arco de San Pedro al final de la calle D. Luís, donde a menudo eran frecuentes "actos escandalosos e de completa desmoralização" 23 . Su estancia en estos lugares, además de darles mala fama, causaba vergüenza y malestar entre los residentes, a pesar de haber sido prohibida la permanencia en la proximidad de las iglesias y las casas de espectáculos, lugares que serían frecuentados por "personas de bien" 24 .

Las inquietudes introducidas por la prensa hacia la prostitución, además de relacionarse con la defensa de las costumbres y de la moral, eran el resultado de la desconfianza que recaía sobre estas mujeres, consideradas transmisoras de la sífilis. ${ }^{25}$ La combinación de estos dos factores, el moralista y el sanitario, fue, sin duda, la justificación de la importancia que la prensa otorgó a las prostitutas y a su conducta. En 1856, el comandante de fuerza milita de infantería situada en el distrito de Viana do Castelo, enfrentándose con la ya mencionada infección de los soldados con el virus sypphitus, solicitó al gobernador civil la toma de medidas. Ordenó que las prostitutas fueran expulsadas del municipio y que las enfermas fueran ingresadas en el Hospital São Marcos de Braga ${ }^{26}$. No era la primera vez que las prostitutas aparecían infectadas de sífilis en la ciudad de Viana do Castelo. En

${ }^{21}$ El día 17 de enero fueron detenidas dos prostitutas en la calle del Marqués por participar en altercados con la vecindad. AHGCVC, $1 .{ }^{a}$ Repartição, G.S.P, 1.13.4.4.-2, sin paginar.

${ }^{22} O$ Vianense, 12 de noviembre de 1861, n. $^{\circ} 448$, sin paginar.

${ }^{23} O$ Vianense, 19 de julio de 1860, n. $^{\circ} 352$, sin paginar; 22 de diciembre de 1864, n. $^{\circ} 1017$ sin paginar; 27 de agosto de 1868, n. $^{\circ} 1567$, sin paginar.

${ }^{24}$ Véase Código Administrativo de 1842. (1849). Coimbra: Imprensa da Universidade, p. 105. Según José Machado Pais, uno de los objetivos de los reglamentos era evitar una posible coincidencia, en el mismo espacio público, de prostitutas y mujeres respetables, especialmente del Regulamento Policial das Meretrizes e Casas Toleradas da Cidade de Lisboa, de 1858. Compárese PAIS, J. (2006). A Prostituição e a Lisboa Boémia. Do século XIX a inícios do século XX..., p. 23.

${ }^{25}$ La relación entre sífilis y prostitución viene de lejos, llegando a la Edad Moderna. Sobre este tema, léase JÜTTE, R. (1996). «Syphilis and Confinement. Hospitals in Early Modern Germany». En Institutions od Confinement, asylums and prisions in Western Europe and North America, 1500-1950. Cambridge: Cambridge University Press, pp. 97-101.

${ }^{26}$ AHGCVC, Registo Geral de Julho de 1856 a 31 de Dezembro, n. ${ }^{\circ} 1.10 .3 .3$, sin paginar 
1839, el administrador del concejo hizo reunir a la mayoría de las prostitutas para ser examinadas, habiendo emitido la Misericórdia de Viana do Castelo carta de guía a las que estaban contagiadas para que se presentasen en el Hospital de São Marcos, de Braga ${ }^{27}$. Por lo tanto, parece innegable que, además de la inmoralidad asociada al ejercicio de la prostitución, el aspecto sanitario contribuye a que la discriminación social que recaía sobre las prostitutas, adquiera en el siglo XIX, nuevos rasgos ${ }^{28}$.

Además, desde el punto de vista conductual, podemos incluir un conjunto de enfermedades que provocaban que las víctimas de las mismas cargasen con el peso del estigma. Era el caso de la tiña, por ejemplo. En el vocabulario cotidiano de las gentes del siglo XIX, encontramos el insulto "tiñoso", utilizado en tono peyorativo, para designar al individuo portador de tiña, enfermedad dermatológica que afectaba a la cabeza y que era contraída, sobre todo, por personas de pocos posibles (pobres), con particular incidencia en los niños ${ }^{29}$. Algunos enfermos de tiña, o sus familiares, especialmente los padres, a causa de su pobreza, se veían obligados a pedir auxilio a las instituciones de beneficencia, como las Misericórdias, para poder llevar a cabo los oportunos tratamientos. Incluso en la década de los 70 del siglo XIX, más concretamente en 1874, la Misericórdia de Viana do Castelo establecía, en el reglamento del hospital que administraba, la no admisión de los enfermos de $t i \tilde{n} a^{30}$.

Especialmente a finales del siglo XVIII y comienzos del XIX, el discurso higienista de las autoridades atribuía a los pobres la responsabilidad por la proliferación de ciertas enfermedades debido a la falta de higiene y la resistencia frente a una política sanitaria destinada a combatir las enfermedades endémicas

${ }^{27}$ ADVC, Administrador del concejo de Viana do Castelo, Copiador Geral -1838, n. ${ }^{\circ}$ 5.16.4.17, sin paginar.

${ }^{28}$ Léase Gómez Bravo, G. (2007). «El delito y la enfermedad en la España del cambio de siglo». Cuadernos de Historia Contemporanéa, vol. Extraordinario, pp. 114-116.

${ }^{29}$ Sobre la repercusión de la tiña en los niños, consúltese ARAÚJO, M. (2008). «Pequenos e pobres: a assistência nas Misericórdias portuguesas da Idade Moderna». En ARAÚJO, M.; FERREIRA, M. (Orgs.). A infância no universo assistencial da Península Ibérica (sécs. XVI-XIX). Braga: Instituto de Ciências Sociais, pp. 141-146. Sobre el auxilio prestado por la Misericórdia de Évora a los niños enfermos de tiña, véase PARDAL, R. (2007). «A Criação dos filhos dos pobres e dos tinhosos: um aspecto esquecido da assistência da Misericórdia de Évora no século XVIII». En Congresso Internacional de História. Territórios, Culturas e Poderes. Actas, vol. II. Braga: Núcleo de Estudos Históricos, Universidade do Minho, pp. 757-766.

30 Archivo Histórico de la Santa Casa de la Misericórdia de Viana do Castelo, (en adelante, AHSCMVC), Regulamento do Hospital da Santa Casa da Misericórdia de Viana do Castelo, n. ${ }^{\circ}$ 4.85.1.6-2, sin paginar. 
como el cólera y la fiebre amarilla ${ }^{31}$. El agua se convierte en un elemento esencial para la limpieza de calles, casas y los cuerpos. La higiene no se relaciona sólo con el simple aseo personal o con exigencias de carácter estético, sino también con la salud. Aunque las clases populares revelaban ya cierta preocupación por la higiene y el aseo del cuerpo, e incluso por la ropa ${ }^{32}$, todavía los cuidados del aseo se limitaban a las partes visibles del cuerpo y al cambio de ropa, focalizándose solo en el aspecto exterior ${ }^{33}$.

En el siglo XIX bajo la influencia de los hábitos burgueses, el baño y otras prácticas de higiene íntima se transforman expresiones de civilización (cultura), idéntificándose la suciedad con la pobreza y la miseria. ${ }^{34}$ Así que podemos afirmar que aunque la teoría no es del todo acorde con la práctica, había una asimilación de conceptos, confirmada por el rechazo de la suciedad, no sólo físicamente sino también moralmente, ya que se ridiculizaba con el propósito de inculcar un conjunto de valores y fortalecer y promover prácticas contrarias a las censuradas. Por lo tanto, burlarse de la falta de limpieza era no sólo una denuncia, sino también un modo estimular a la gente a cuidar su aseo. Las grandes epidemias que marcaron a Europa durante el siglo XIX hicieron emerger un nuevo discurso higienista y llevaron a la asimilación y generalización de un conjunto de conceptos para el desarrollo de nuevos patrones de comportamiento. Se continúa buscando a los responsables de la propagación de la enfermedad, aplicándose la política de saber para controlar.

Este principio es más difícil de aplicar cuando nos referimos a la enfermedad mental. La humanización de la locura, fruto del desarrollo científico, permitió que se abordará desde la perspectiva de la racionalidad y no del oscurantismo. Este cambio tendrá consecuencias en el modo de considerar de los enajenados.

${ }^{31}$ Sobre la importancia de la higienización de las clases populares, léase VIGARELLO, G. (1988). o Limpo e o Sujo. A Higiene do Corpo desde a Idade Média. Lisboa: Editorial Fragmentos, pp. 151157.

32 AHGCVC, Moléstias - Arcos de Valdevez - Correspondência do Administrador do Concelho, n. ${ }^{\circ}$ 1.13.6.12-8, sin paginar; AMMço, Administração do Concelho. Ofícios e Circulares remetidos pelo Governador Civil - 1848, n. ${ }^{\circ}$ 1.3.1.4-2, sin paginar. AHGCVC, Cholera Morbus, n. ${ }^{\circ}$ 1.13.6.12-6, sin paginar.

${ }^{33}$ En la Era Moderna se pensaba que la ropa era portadora de higiene y este concepto era puesto en práctica cuando se realizaba la muda de ropa ya que esta absorbía los sudores. De este modo, se creía que la ropa, por sí misma, lavaba y limpiaba el cuerpo. Vigarello, G. (1988). O Limpo e o Sujo. A Higiene do Corpo desde a Idade Média..., pp. 53-66.

${ }^{34}$ Sobre la responsabilidad de los pobres en la propagación de epidemias a lo largo del siglo XIX, léase Rodrigues, J. (1884). «Lisboa e a Cholera». Biblioteca do Povo e das Escolas, n. ${ }^{\circ}$ 88, p. 24; CARdoso, J. (1912). «Micróbios e doenças». Biblioteca do Povo e das Escolas, n. ${ }^{\circ}$ 152, p. 52. 
Progresivamente, deja de ser observado como un obstáculo para el progreso social, para ser considerado un enfermo con perspectivas de curación a través de la aplicación de un tratamiento adecuado. Sin embargo, esto no fue una proceso rápido, lineal y generalizado. Incluso podemos decir que estas dos concepciones de la locura convivirán en la sociedad occidental durante mucho tiempo. En el siglo XIX, los locos seguían siendo considerados aún como un obstáculo que ponía en peligro, por su comportamiento, los valores de la sociedad burguesa. Considerado desprovisto de racionalidad, no podía considerarse un hombre en el verdadero sentido de la palabra, no gozando, por tanto, de libertad. Como resultado de su desvío, constituía un ataque a la propiedad y la paz pública, era considerado un vago y, a veces, presentaba síntomas que lo aproximaban al fanatismo religioso. Era el "idiota", el "loco" el "demente", tal y como aparece descrito en documentos del XIX.

Así, la sociedad del siglo XIX irá lidiando con los hombres y mujeres demenciados de forma claramente antagónica: unas veces considerándolos enfermos, otras como un obstáculo para el progreso social. Admitía que el loco debe ser ayudado, pero también exigía protección contra los riesgos que su presencia implicaba. La medida ideal par combatir, eliminar y, si era posible, tratar al alienado, era el aislamiento. El internamiento no debía ser entendido como un acto deshumanizado sino más bien como un paso crucial para el desarrollo de la sociedad, a través del aislamiento de sus individuos menos capaces, a pesar de la visión más humanista que se desarrolló durante el siglo XVIII sobre las enfermeddes y los enfermos mentales.

El aislamiento del paciente podía concretarse dentro de la propia familia, a través de su aislamiento en una habitación de la casa. Sin embargo, no siempre había condiciones para hacer esto posible. Las dificultades no eran sólo económicas, sino que también tenían que ver con la propia manifestación de la locura a través de comportamientos más o menos agresivos, lo que originaba miedo e impotencia en las personas más cercanas. A veces, las autoridades sólo tenían conocimiento de su existencia cuando se producían actos violentos, o cuando las familias, debido a la falta de medios para mantenerlos, reclamaban la intervención y ayuda de las autoridades.

En otros casos, los alienados, desprovistos de recursos materiales y sin el apoyo de la familia, eran entregados a su suerte y deambulaban por las calles y caminos, inquietando a la población. Algunos de ellos mendigaban o atacaban a los transeúntes y todo lo que estuviese a su alcance, otros tenían actitudes menos apropiadas, por lo que tenían que ser apartados del contacto con los demás. En estas situaciones, su aislamiento se hizo aún más apremiante y el destino era 
entonces la cárcel o el hospital. Sin embargo, estas dos instituciones siguen considerándolo persona non grata. El internamiento de los enfermos mentales en las cárceles no era impuesto únicamente por la enfermedad que sufrían, sino también por las conductas que, de alguna manera, alteraban el orden público y la seguridad $^{35}$.

En general, los hospitales se negaban a recibir a los enfermos mentales, alegando hacinamiento y falta de condiciones para ofrecerles asistencia. Cuando se les acogía en los hospitales generales, el tratamiento que se les proporcionaba difería poco del aplicado en la cárcel, que no iba más allá del simple encarcelamiento, sin que se les suministrase ningún tipo de tratamiento específico $^{36}$. El único beneficio concedido por algunos hospitales era el retiro en un espacio individual, mientras que en la cárcel permanecían en salas comunes, convirtiéndose en motivo de burla para los restantes presos. ${ }^{37}$.

El internamiento de locos en Europa data de finales del siglo XVI. Estaban presentes en los asilos, correccionales y en los hospitales generales ${ }^{38}$. Formaban parte de una masa indiferenciada de personas, entre las que se incluían los mendigos, vagabundos, prostitutas, pequeños delincuentes, que era enviada a estos lugares sin recibir ningún tratamiento específico. Un siglo más tarde, se reconoció la importancia del internamiento del enfermo mental. Ya en el siglo XIX, constatamos que países como Inglaterra, Francia e Italia disponían ya de

35 En el siglo XIX, había todavía dificultad para una definición de locura, ya que esta variaba según la opinión de los médicos, no existiendo una idea uniforme sobre las causas que la originaban. Compruébese Chevalier, L. (2007). Classes laborieuses et classes dangereuses. Paris: Éditions Perrin, pp. 334-338.

${ }^{36}$ La "falta de condiciones" fue el argumento utilizado sucesivamente para no ingresar a los dementes en hospitales.

${ }^{37}$ Véase el caso paradigmático de José da Lomba, quien, debido a su locura crónica, de vez en cuando atacaba a los habitantes de Viana do Castelo. En 1836, dada la falta de disponibilidad del hospital de la Misericordia para acogerlo, fue, preventivamente, encarcelado en la prisión local, siendo solicitado con posterioridad su traslado al hospital de San José. Archivo nacional de la Torre do Tombo (en adelante ANTT), Ministério do Reino, Ofícios recebidos, Maço 2104, sin paginar.

38 Léase Pichot P.; Fernandes, B. (1984). Um Século de Psiquiatria e a Psiquiatria em Portugal. Lisboa: Roche, p. 18. El internamiento de los enfermos mentales se va efectuando a medida que la locura va dejando de ser considerada el resultante de factores externos al individuo, especialmente de tipo esotérico, y pasa a explicarse racionalmente, desde el punto de vista patológico, como una enfermedad. Compruébese Alvarez-URía, F. (1983). Miserables y locos. Medicina mental y orden social en la España del siglo XIX. Barcelona: Tusquets editores, Cuadernos Ínfimos, pp. 2627. Sobre la asistencia prestada a los dementes en España, en el siglo XIX, léase CARASA SOTO, P. (1987). «Cambios en la tipología del pauperismo en la crisis el antiguo régimen». Investigaciones históricas: Época moderna y contemporánea, n. ${ }^{\circ}$ 7, pp. 143-144. 
instalaciones apropiadas para el cuidado de los enfermos mentales. En Portugal, los más pobres eran condenados a la reclusión, aunque algunos, agraciados por la suerte, fuesen ingresados en los hospitales. Los de familia más acomodada eran confinados en los propios hogares donde pasarían el resto de su vida ${ }^{39}$.

Efectivamente, los locos eran rechazados por la sociedad y las instituciones. Sólo a partir de 1818, los alienados comenzaron a ser recibidos en gran número en el Hospital San José de Lisboa. Fue precisamente a este hospital al que, hasta 1848, eran enviados los enfermos mentales del distrito de Viana do Castelo ${ }^{40}$. El Hospital de Santo António, en Oporto, también recibía estos enfermos, pero sin imponer criterios específicos de admisión. La inestabilidad política y las dificultades económicas del país, que se vieron agravadas por la guerra civil, llevó a la postergación de la construcción de manicomios en Portugal. Después de 1848, los pacientes comenzaron a ser transferidos al Hospital de Rilhafoles, el trabajo de los sacerdotes en el antiguo convento de S. Vicente de Paúl, en Lisboa. Esta es la primera casa de locos portugueses, más bien, el asilo, que aparece consagrado en sus estatutos $^{41}$. Preveía el aislamiento y la clasificación de los pacientes, así como su ocupación en labores agrícolas y otros oficios. Aceptaba dementes de ambos sexos y adoptaba un sistema basado en el castigo y la recompensa.

Para ser admitido en aquella unidad hospitalaria, era necesario que el paciente presentase un certificado médico que avalase su estado de alienación, evitando de este modo el internamiento de personas sanas, y una guía privada o de la institución que se responsabilizaba del pago del tratamiento. De acuerdo con lo dispuesto en el Reglamento de la institución, la admisión de los enfermos podía ser solicitada por la familia o decidida por las autoridades competentes. En todos los casos examinados, el envío de pacientes por el gobernador civil contaba con el apoyo del administrador del concejo, que, a su vez, se apoyaba en certificados médicos ${ }^{42}$.

39 Véase Pereira, P.; Gomes, E.; Martins, O. (2005). «A Alienação no Porto: o Hospital de Alienados do Conde Ferreira (1883-1908)». Revista da Faculdade de Letras. História, III serie, vol. 6, p. 99.

${ }^{40}$ Sobre la falta de condiciones del Hospital de San José para acoger a los enfermos mentales procedentes de todo el país, léase PereirA, A. (1986). "A institucionalização da loucura em Portugal». Revista Crítica de Ciências Sociais, n. ${ }^{\circ} 21$, pp. 87-88.

${ }^{41}$ Véase Regulamento do Hospital d'Alienados estabelecio no Edificio de Rilhafoles. (1851). Lisboa: Imprensa Nacional, p. 59.

${ }^{42}$ Según el Código Administrativo de 1842, correspondía al administrador del concejo evitar el periplo (peregrinaje) de los enfermos mentales a través de las localidades de su jurisdicción. Cuando eran apresados y enviados al hospital, primero al de San José y después al de Rilhafoles, debían ir 
Además en la década de los 60, problemas como el hacinamiento, la presencia de enfermos incurables y la falta de personal médico comenzaron a afectar al Hospital Rilhafoles. Por otro lado, se rumoreaba que no se cumplían los requisitos de ingreso de los pacientes y que no se les trataba con dignidad. En 1862, la situación se agravó de tal manera que se organizó un comité para evaluar su gestión. Con los años, este área hospitalaria se fue convirtiendo en un espacio destinado, especialmente, a enfermos pobres, sin familia, que había sido detenidos por la policía y remitidos a las autoridades administrativas, lo que explica el problema del hacinamiento,que causaba trastornos en su funcionamiento, especialmente en materia de higiene y atención médica.

La cárcel y el hospital general continuaban siendo el destino principal de los locos. El llamado "loco manso" continúa en la calle, pero el "loco violento" era enviado a prisión. Este era el panorama en el ámbito de la protección de las enfermedades mentales, a pesar de que, en 1889, se promulgó la primera ley de atención psiquiátrica, la "Lei de Sena", que preveía la creación de cuatro hospitales más y de enfermerías anexas a las cárceles, exámenes médicos obligatorios para los autores de los delitos que mostraran síntomas de alienación e incluso la creación de un fondo de beneficencia pública para los alienados. Sin embargo, esta ley no se aplicó plenamente debido a las dificultades financieras que afectaban al país.

A finales del siglo XIX, los enfermos mentales del Alto Miño siguen siendo enviados al Hospital de Rilhafoles. Desconocemos las razones que llevaban a las autoridades, después de 1883, a enviarlos a aquella unidad hospitalaria, teniendo una mucho más cerca, en la ciudad de Oporto. Probablemente, este procedimiento podría ser debido a la masificación del Conde Ferreira, especialmente en lo que respecta a los pacientes jubilados. El envío de los locos para las dos unidades hospitalarias seguía siendo un proceso lento, causado por el problema ya mencionado de la masificación, sometiéndolos a una larga espera por una vacante, después de haber cumplido todos los requisitos. La aparición de estos hospitales psiquiátricos en Portugal en el siglo XIX resultó ser una señal de cambio en la percepción de la locura, que pasó a ser considerada, desde el punto de vista científico, como una enfermedad mental, a la que se intentó dar respuesta, en términos sociales, con la creación de instituciones especializadas para su tratamiento. Conviene señalar que el ingreso en "hospitales para enfermos mentales" podía no significar el fin de los problemas. Esto se debe a que algunos

acompañados de un certificado del médico que los examinó, debidamente firmado por el administrador del concejo, solo así el enfermo era admitido en el hospital. Compruébese Código Administrativo..., p. 108. 
internos huían de la institución y otros, agotado el plazo para el tratamiento, eran dados de alta sin estar curados, por lo que su reingreso era inevitable ${ }^{43}$. Es importante mencionar también a los que acababan muriendo en el aislamiento del hospital, lejos de la familia y de su tierra natal ${ }^{44}$. Hubo varios casos de muerte de pacientes de las tierras altas de Miño, pero de uno solo de ellos se especifica la causa de la muerte..

La hospitalización de los pacientes bien podía limitarse a un periodo preestablecido como prolongarse durante varios años o hasta que se produjese la muerte. Debido a la escasez de información no siempre es posible determinar con precisión el tiempo de permanencia de los enfermos en estas instituciones. Entre 1892 y 1893, cuando Miguel Bombarda asumió la dirección del Hospital de Rilhafoles, y entre 1899 y 1900, esta institución recibió un mínimo de seis y un máximo de 11 personas naturales en el distrito de Viana do Castelo

Es difícil trazar el camino que estos hombres y mujeres recorrieron antes de ser afectados por la enfermedad. Muchos tenían una ocupación como jornalero, albañil, panadero, cestero, soldado, criada, dependiente o labrador. Si algunos lograban formar una familia, siendo afectados por la enfermedad en una etapa más avanzada de sus vidas, otros, cuya enfermedad se había manifestado tempranamente y permanecían solteros, continuaban en la casa familiar, habitualmente en la de los padres. Según Antonio María de Sena, "los idiotas en general, no se casan, a menos que sean muy ricos, y son muy pocos"

Al mismo tiempo, la locura era considerada causa de empobrecimiento. Algunos enfermos mentales eran retratados como derrochadores, gastadores y dominados por impulsos incontrolables que les llevaba a destruir sus propios bienes. En el caso de las mujeres, la situación podía ser, incluso, más grave, dado que, a veces, eran simplemente abandonadas por sus maridos, quedando sin medios que garantizasen su sustento.

En un momento en el que las distancias eran más grandes y el apoyo a los enfermos mentales casi inexistente, la locura en sí misma era un problema social,

${ }^{43}$ En abril de 1873, José Maria Quintães, natural de Vila Nova de Cerveira huyó de Rilhafoles. AHGCVC, Assistência Social e Saúde Pública. Correspondência relativa a alienados., n. ${ }^{\circ} 1.16 .6 .1$ 15 , não paginado.

${ }^{44}$ Este fue el caso de Domingos José Esteves, que fue admitido en Rilhafoles en 1880, donde falleció cinco años después. AHGCVC, Assistência Social e Saúde Pública. Correspondência relativa a alienados., n. ${ }^{\circ}$ 1.16.6.1-1, sin paginar.

${ }^{45}$ Léase SENA, A. (2003). Os alienados em Portugal. Lisboa: Ulmeiro, p. 262. 
que afectaba principalmente a los estratos más necesitados de la sociedad, contribuyendo al empeoramiento de las condiciones de vida de aquellos que, de alguna manera, estaban afectados por la enfermedad. La situación se complicaba aún más cuando se asociaba a la ausencia de un marco familiar, una edad avanzada, entre otros factores. En el caso particular del distrito de Viana do Castelo, las autoridades administrativas, en cumplimiento de la legislación vigente, procuraban impedir que los alienados vagasen por los espacios públicos, enviándolos al Hospital de Rilhafoles y, más tarde, al de el Conde Ferreira. Sin embargo, como ya dijimos, no siempre el ingreso en estas instituciones fue bien aceptado, por lo que la cárcel y la calle seguía siendo la morada, aunque temporal, de muchos de estos pacientes.

Nuestro estudio considera solo a los enfermos mentales que, a causa de su pobreza o comportamiento agresivo, requerían la intervención de las autoridades administrativas. Teniendo en cuenta el distrito de Viana do Castelo, en el censo de 1878 se señalaba la existencia de 461 locos e "idiotas", con 242 hombres y 219 mujeres $^{46}$. Como podemos imaginar, no todo el mundo se beneficiaría de la atención médica. Entre finales de 1881 y 1882, Antonio María de Sena, en la preparación para la apertura del Hospital Conde Ferreira, decidió enviar un oficio a los médicos de los municipios y a los gobernadores de los distritos con el fin de obtener una descripción(mapa) de la locura en diferentes partes del país. Viana do Castelo, Ponte de Lima, de Caminha eran los municipios que, entonces, más enfermos mentales registraban, destacando este último por presentar "más alto coeficiente de alienación", con un loco por cada $413^{47}$. Antonio María de Sena apuntaba la existencia de un total de 323 locos en esta zona del país, siendo en su mayoría pobres $(70 \%)$ y solteros $(80 \%)$.

A pesar de las dificultades expuestas, no es descabellado decir que se lograron algunos éxitos en la forma de encarar y cuidar la enfermedad mental . Uno de ellos consiste en la afirmación, ya en el ámbito científico, de que el loco es un enfermo. Como tal debía ser tratado, por lo que se hacía necesario desarrollar mecanismos de asistencia que pasaban, especialmente, por la creación de instituciones de acogida dotadas con los medios adecuados. Sin embargo, esta fue una conclusión tardía en el contexto portugués. Por tierras lusas, los grandes estudiosos de la locura, las monografías gnoseológicas y los primeros espacios dedicados a la acogida de locos surgió en el siglo XIX. También es cierto que a finales de este siglo, algunos aspectos de la locura eran aún intangibles, lo que explica el hecho de que en las

\footnotetext{
${ }^{46}$ Sobre este tema, consúltese SENA, A. (2003). Os alienados em Portugal..., p. 213.

${ }^{47}$ Véase SEnA, A. (2003). Os alienados em Portugal..., p. 228.
} 
instituciones de salud mental siguiesen existiendo auténticas cárceles, llamadas "quartos fortes", donde eran enviados los pacientes "más agitados"48. Así se entiende que, a finales del siglo XIX, António Maria de Sena haya tachado al Hospital de Rilhafoles de"deposito desordenado de infelices dementes que no tienen medios de subsistencia" 49 .

La tuberculosis era un azote que atacaba a una parte significativa de la población. La medicina portuguesa, con escasos recursos farmacológicos para combater la enfermedad, reforzó las medidas que se consideraban eficazes, es decir, la prevención y el aislamiento. En este sentido, se crearon hospitales y sanatorios, que, a partir de mediados del siglo XIX, asumieron un importante papel en la lucha contra la tuberculosis; se implantaron reglas sociales con el objetivo de mejorar la condición física y mental de la población, y se apostó por la formación de médicos en el extranjero en el área de neuma-tisiología. Mientras, en todo el país, iban surgiendo sanatorios para el tratamiento de la tuberculosis.

Sin embargo, a finales del siglo XIX, la tuberculosis todavía alcanzaba grandes proporciones en Portugal, lo que llevó a la creación en 1899 de la Asistencia Nacional de Tuberculosos, que, entre otros objetivos, pretendía promover la construcción de hospitales marítimos, sanatorios en lugares apropiados e incluso hospitales para tuberculosos a los enfermos incurables ${ }^{50}$.

También se decidió la implantación en todas las capitales de distrito de institutos para el tratamiento de la tuberculosis y, por iniciativa de Miguel Bombarda, se estableció la Liga Portuguesa contra la Tuberculosis. Este conjunto de medidas no impidó que, a principios del siglo XX, esta enfermedad continuase afectando a todos los estratos sociales, con una mayor incidencia en las clases más bajas como resultado de sus precarias condiciones de vida.

En los últimos años de la monarquía, las actas de la Misericordia de Viana do Castelo daban cuenta de la intención de proceder a la creación de un hospital de reposo para los pacientes con enfermedades pulmonares. Mientras tanto, entre 1901

\footnotetext{
${ }^{48}$ Léase SENA, A. (2003). Os alienados em Portugal..., pp. 150-151.

${ }^{49}$ Compárese SEnA, A. (2003). Os alienados em Portugal..., p. 170.

${ }^{50}$ Acerca de esta institución, léase MARTINS, A. (1999). Génese, emergência e institucionalização do serviço social portugués. Lisboa: Fundação Calouste Gulbenkian, Fundação para a Ciência e Tecnologia, pp. 53-55.
} 
y 1911, se construyeron algunos edificios de asistencia a enfermos de tuberculosis: dos sanatorios marítimos, uno terrestre y cinco dispensarios ${ }^{51}$.

El 19 de marzo de 1907, murió Roberto Gonçalves Barreiros, dejando todo su patrimonio a la Santa Casa da Misericordia de Viana do Castelo y a la Congregación de la Caridad. Era parte de la finca llamada la Quinta de Valverde, situada en São João d'Arga, uno de los puntos más altos del distrito de Viana do Castelo. La mitad perteneciente a la Congregación de la Caridad fue adquirida en subasta pública en 1908 por la Misericordia de Viana con el objetivo de crear un establecimiento especializado en el tratamiento de las enfermedades infecciosas.

Con la caída de la monarquía, el establecimiento de la República y Portugal sumida en una profunda crisis económica, los pobres, desnutridos y físicamente debilitados, fueron los más afectados por las enfermedades infecciosas, incluida la tuberculosis. El azote de la enfermedad aparecía plasmado en las páginas de los periódicos que publican los remedios caseros que supuestamente garantizaban la cura de la enfermedad. Lo cierto es que entre 1910 y 1920, la tuberculosis había afectado a más de 100 mil portugueses. Años más tarde, ya en 1913, el ayuntamiento de Viana do Castelo autorizó la modificación del edificio de la quinta Valverde y al año siguiente, el Servicio Nacional de Asistencia concedió una subvención por la cantidad de $700 \$ 00$ para la terminación de las obras y la adquisición de equipo y mobiliario para el hospital, que, según las fuentes, estaría destinado al tratamiento de enfermos tuberculosos ${ }^{52}$. En Portugal, los principales mecanismos de lucha contra la tuberculosis fueron institucionales: hospitales, sanatorios, dispensarios y preventorios.

El padre Luis da Cunha Sotomaior Faria, fallecido en 1918, había nombrado heredera a la Misericordia de Viana do Castelo. Era su voluntad que su legado se destinase al hospital para tuberculosos, al centro de lactancia, o la creación de la oficina de S. José ${ }^{53}$. Finalmente, en 1924, la Mesa de la Misericordia decidió acelerar lo más posible las obras del hospital para reposo de enfermos tuberculosos, acordando que se adquiriese el equipamiento necesario para abrir las puertas. En el último día de 1928, la Misericordia ordenó que el hospital fuese abierto, comenzando a funcionar en el mes de enero del año siguiente.

\footnotetext{
${ }^{51}$ La ciudad de Porto construyó su primer Dispensario antituberculoso a principios del siglo XX y vio surgir su primer sanatorio en 1926, al amparo de la Santa Casa de la Misericórdia de aquella ciudad. Véase Ferreira, M. (2005). A doença do peito. Contributo para o estudo histórico da tuberculose..., p. 20.

52 AHSCMVC, Processo relativo à construção do hospital - $3.27 .3 \mathrm{cx} .2-30$, fl. 1.

53 AHSCMVC, Processo relativo à construção do hospital - 3.27 .3 cx. 2-30, fl. 1.v.
} 
En el mismo año, se propusieron obras de mejora y ampliación de la estructura inicial. Sin embargo, estas resultaron insuficientes, dejando al descubierto innumerables carencias de la institución, especialmente en lo que respecta a las condiciones de las enfermerías y salas de curas. De hecho, en 1832, el director de la clínica manifestó su apoyo al cierre temporal del hospital. Tras este dictamen, fueron dados de alta todos los pacientes, a excepción de dos, para iniciar obras estructurales, dada la obsolescencia del edificio.

Sin embargo, cabe señalar que a pesar de la incidencia de la tuberculosis en el distrito de Viana do Castelo, era muy reducido el número de pacientes que acudían al Hospital Luis Faria, en el que prestaban servicio de tres a cuatro enfermeras, que fueron retiradas más tarde, haciendo imposible su funcionamiento. En 1933, los médicos reconocieron que no disponían de las condiciones adecuadas para continuar en activo, ya que se estaba convirtiendo en un auténtico depósito de cadáveres, en el que prácticamente ninguno de los pacientes se curaba y todos fallecían ${ }^{54}$.

Por otra parte, el Hospital Luís Faria no era el más apropiado para asistir tuberculosos y los ingresos de los legados eran insuficientes para iniciar obras de adaptación ${ }^{55}$. Por esta razón, se empezó a replantear su finalidad, siendo admitida la posibilidad de redirigir la actividad a los hijos de enfermos de tuberculosis en peligro de contagio ${ }^{56}$. Los niños eran considerados un grupo de riesgo y, como tal, comenzaron a ser ubicados en preventorios, que funcionaban tanto como guarderías como lugares que permitían prevenir la tuberculosis. Sin embargo, este proyecto fue rechazado por el personal médico debido a la escasez de instalaciones y la falta de condiciones. Sin embargo, la opinión de los médicos no satisfizo a la Misericordia local, empeñada en la transformación del sanatorio en un preventorio destinado a acoger los niños pobres en riesgo de contagio ${ }^{57}$. Este compromiso se desvaneció, por lo que, en 1837, el provedor de la Misericordia de Oporto preguntó a su homólogo de Viana do Castelo sobre el legado del Padre Luis Faria, instando a la transformación del sanatorio en un dispensario de leche. Lo cierto es que el hospital estaba cerrado desde mayo de 1833 y había que darle un destino.

En Portugal, en algunos casos, los hospitales fueron adaptados para recibir pacientes tuberculosos, cuyo tratamiento se basaba en una cuidada alimentación,

\footnotetext{
${ }^{54}$ AHSCMVC, Processo relativo à construção do hospital - $3.27 .3 \mathrm{cx} .2-30$, fl. $5 \mathrm{v}$.

55 AHSCMVC, Processo relativo à construção do hospital - 3.27.3 cx. 2-30, fl. 15

${ }^{56}$ AHSCMVC, Processo relativo à construção do hospital - $3.27 .3 \mathrm{cx} .2-30$, fl. 6 .

57 AHSCMVC, Processo relativo à construção do hospital - $3.27 .3 \mathrm{cx} .2-30$, fl. 9 .
} 
aire puro y mucho descanso. Pero, como ocurrió en otras partes de Europa, también fue necesaria la construcción de nuevos hospitales, en lugares apropiados para dar cabida, tan sólo, a este tipo de pacientes.

La vacuna B.C.G., a pesar de haber sido aplicada por primera vez en 1921, se incluyó en el plan de vacunación en Portugal tan solo a partir de 1928, curiosamente como resultado de la actuación de un médico vianense $\mathrm{e}^{58}$. En estos momentos, el distrito de Viana do Castelo contaba ya con un sanatorio marítimo, el llamado Sanatório da Gelfa, construido entre 1910 e 1911, destinado a enfermos que padecían tuberculosis ósea ${ }^{59}$.

La apertura de sanatorios, además de revelar el carácter público que asumió la lucha contra la tuberculosis, fue el resultado no sólo de la necesidad de proporcionar a los pacientes los tratamientos adecuados, sino también de aislarlos del resto de la población y evitar así la propagación de la enfermedad ${ }^{60}$. Pronto, este será el objetivo principal de estos lugares, quedando la perspectiva de mejora del paciente relegada a un segundo plano

Los ejemplos de enfermedades abordados aquí -la sífilis, la tiña, la locura y la tuberculosis- llevaban, por una razón u otra, a la aparición de distintas formas de discriminación o segregación, consentidas o forzadas, más visibles u ocultas. Sin embargo, estas actitudes no se limitaban a una minoría sino que refleja un sentimiento generalizado, provocado e impulsado a menudo por las medidas adoptadas por los propios Estados, que, a su vez, actuaban con la visión que la medicina tenía entonces de de ciertas enfermedades y enfermos.

${ }^{58}$ Compárese PARREIRA, L. (1953). «Vacinação pela BCG nas escolas primárias». Jornal Médico, vol. 22 , n. ${ }^{\circ} 557$, pp. 633-647.

59 Véase Ferreira, M. (2005). A doença do peito. Contributo para o estudo histórico da tuberculose..., p. 43.

60 Sobre este tema, consúltese BYRNE, K. (2011). Tuberculosis and the Victorian Literary Imagination. Cambridge: Cambridge University Press, p. 178. 